\title{
Sensory and Working Memory Representations of Small and Large Numerosities in the Crow Endbrain
}

\author{
Helen M. Ditz and Andreas Nieder \\ Animal Physiology Unit, Institute of Neurobiology, University of Tübingen, 72076 Tübingen, Germany
}

Neurons in the avian nidopallium caudolaterale (NCL), an endbrain structure that originated independently from the mammalian neocortex, process visual numerosities. To clarify the code for number in this anatomically distinct endbrain area in birds, neuronal responses to a broad range of numerosities were analyzed. We recorded single-neuron activity from the NCL of crows performing a delayed match-to-sample task with visual numerosities as discriminanda. The responses of $>20 \%$ of randomly selected neurons were modulated significantly by numerosities ranging from one to 30 items. Numerosity-selective neurons showed bell-shaped tuning curves with one of the presented numerosities as preferred numerosity regardless of the physical appearance of the items. The resulting labeled-line code exhibited logarithmic compression obeying the Weber-Fechner law for magnitudes. Comparable proportions of selective neurons were found, not only during stimulus presentation, but also in the delay phase, indicating a dominant role of the NCL in numerical working memory. Both during sensory encoding and memorization of numerosities in working memory, NCL activity predicted the crows' number discrimination performance. These neuronal data reveal striking similarities across vertebrate taxa in their code for number despite convergently evolved and anatomically distinct endbrain structures.

Key words: corvid songbird; endbrain; number; single-unit recordings

\section{Significance Statement}

Birds are known for their capabilities to process numerical quantity. However, birds lack a six-layered neocortex that enables primates with numerical competence. We aimed to decipher the neuronal code for numerical quantity in the independently and distinctly evolved endbrain of birds. We recorded the activity of neurons in an endbrain association area termed nidopallium caudolaterale (NCL) from crows that assessed and briefly memorized numerosities from one to $30 \mathrm{dots}$. We report a neuronal code for sensory representation and working memory of numerosities in the crow NCL exhibiting several characteristics that are surprisingly similar to the ones found in primates. Our data suggest a common code for number in two different vertebrate taxa that has evolved based on convergent evolution.

\section{Introduction}

Over the past decade, the neuronal code for numerical quantity representations has been studied intensively in human and nonhuman primates, identifying the associative neocortices, the parietal and frontal lobes, as key areas in number representations (Nieder, 2013). But how is numerical information represented in vertebrates that lack a six-layered neocortex? Birds show elaborate quantification skills (Bogale et al., 2011; Scarf et al., 2011;

\footnotetext{
Received May 10, 2016; revised Sept. 9, 2016; accepted Sept. 12, 2016.

Author contributions: A.N. and H.M.D. designed research; H.M.D. performed research; H.M.D. analyzed data; H.M.D. and A.N. wrote the paper.

This work was supported by the Deutsche Forschungsgemeinschaft (Grant NI 618/3-1 to A.N.).

The authors declare no competing financial interests.

Correspondence should be addressed to Andreas Nieder, Animal Physiology Unit, Institute of Neurobiology, University of Tübingen, Auf der Morgenstelle 28, 72076 Tübingen, Germany. E-mail: andreas.nieder@uni-tuebingen.de.

DOI:10.1523/JNEUROSCI.1521-16.2016

Copyright $\odot 2016$ the authors $\quad 0270-6474 / 16 / 3612044-09 \$ 15.00 / 0$
}

Moll and Nieder, 2014), but their cognitive endbrain structures developed from different pallial precursors compared with mammals, which led to a distinct anatomical layout of the avian endbrain (Jarvis et al., 2005; Butler et al., 2011). Recently, we have shown that neurons in the nidopallium caudolaterale (NCL) of crows, an associative endbrain structure in birds (Divac et al., 1985; Güntürkün, 2005), respond systematically to the presentation of small numerosities from one to five items (Ditz and Nieder, 2015).

Although this study provided a first glimpse into number coding in birds, several questions remain unanswered due to the restricted numerosity range from one to five tested so far. First, for small numerosities, two distinct mechanisms are discussed that are difficult to disentangle based on tests with small numerosities (Feigenson et al., 2004): an "object file system" (OFS) that is precise but can only represent up to four items (set-size limit) and an approximate "analog number system" (ANS) that represents small and large numerosities with decreasing precision as magnitudes increase. The ANS is 
A
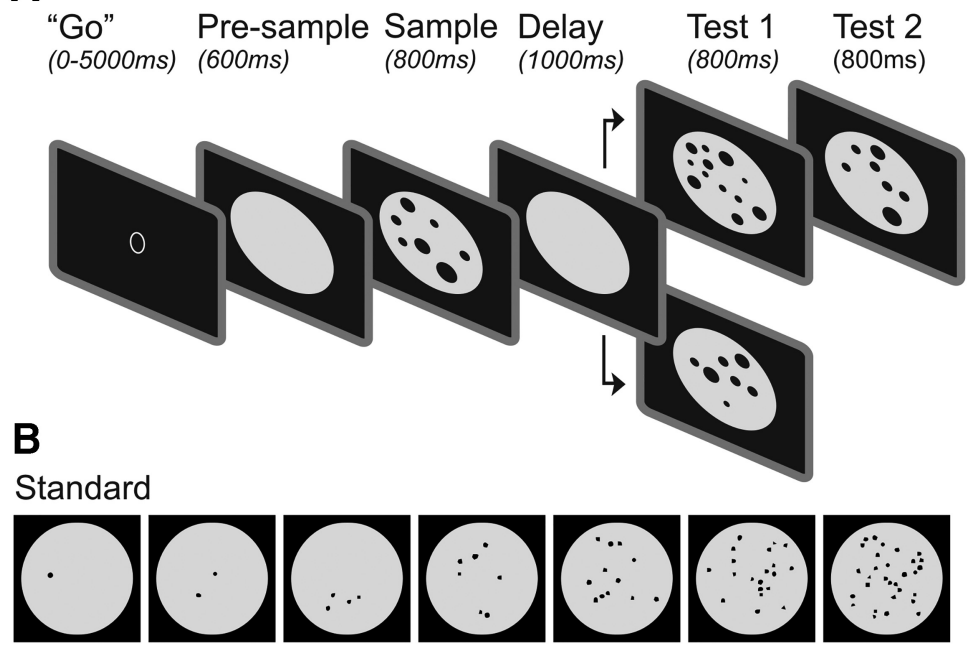

Control (equal area and density)

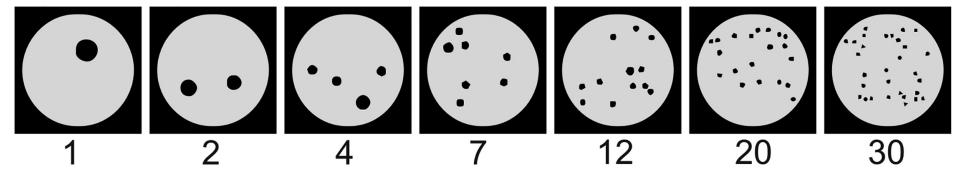

Figure 1. Task protocol and stimuli. $\boldsymbol{A}$, Delayed match-to-numerosity task: the crow initiates a trial by bringing its head into the light barrier when the " $\mathrm{Go}$ " stimulus appears. Afterward, a gray background circle appears. After $600 \mathrm{~ms}$, a pseudorandom sample stimulus is shown for $800 \mathrm{~ms}$, followed by a 1000 ms delay. Then, in $50 \%$ of the cases, a match follows, which contains the same number of dots as the sample stimulus but differs in appearance. If a match appears (match condition), the crow has to respond by moving its head to leave the light barrier to receive a reward. If a non-match appears as test1 (non-match condition), the crow is not allowed to respond (i.e., keep the head still and the light barrier closed) and wait for $800 \mathrm{~ms}$. Afterward, the test 2 stimulus appears, which is always a match, and the crow has to respond by leaving the light barrier to complete the trial successfully. $\boldsymbol{B}$, Example stimuli for both stimulus sets: standard and control stimuli (equating total black area and dot density across numerosities) are shown as examples. The number space between 1 and 30 was covered by 7 numerical values $(1,2,4,7,12,20$, and 30).

in accordance with the Weber-Fechner law, which states that the relationship between a physical stimulus and its perception is of logarithmic nature (Nieder and Miller, 2003; Merten and Nieder, 2009). To determine whether tuned numerosity-selective neurons might be a special feature of the OFS or if they are a signature of numerosity coding for the whole number range, probing the responses of NCL neurons to both small and large numerosities is necessary.

Second, the code for number in the crow NCL is still debatable. According to the idea of "summation coding", quantity is encoded by the neurons' monotonically increasing and decreasing response functions (Meck and Church, 1983; Romo et al., 1999; Roitman et al., 2007). The alternative "labeled-line code" predicts that numerical quantities are encoded by the maximum response rate of a particular neuron, resulting in bell-shaped tuning curves (Nieder and Miller, 2003: Nieder and Merten, 2007; Nieder, 2012). Because tuning curves get easily truncated in a restricted numerosity range from one to five, stimulation with a broad range of numerosities allowing the construction of detailed response functions is required.

Third, the processing of numerical information toward a goal requires that number representations are actively retained after the stimulus ceased. Whether and how NCL neurons constitute a correlate of numerical working memory has not been investigated so far. Given that NCL neurons have been shown to maintain sensory information across temporal gaps (Veit et al., 2014, 2015a, 2015b; Moll and Nieder, 2015), NCL is also a promising structure for numerical working memory.
In the current study, we recorded single-unit activity in the NCL of crows discriminating visual numerosities from one to 30 in a delayed match-to-sample task. We present a detailed analysis of the neurons' responses arguing for a labeledline code that obeys the Weber-Fechner law and is in agreement with the behavioral data (Ditz and Nieder, 2016). The same response characteristics were found during the delay period, suggesting that NCL neurons constitute a neuronal correlate of numerical working memory.

\section{Materials and Methods}

Subjects. Two hand-raised crows (Corvus corone corone), one male and one female, were trained on a delayed match-to-sample task with the number of items in dot displays as discriminative stimuli. All crows were obtained from the institute's breeding facilities. The crows were housed in social groups in spacious indoor aviaries (Hoffmann et al., 2011). During the sessions, they were maintained on a controlled feeding protocol and earned food during and after the daily tests. All animal preparations and procedures complied with the National Institutes of Health's Guide for the Care and Use of Laboratory Animals and were approved by the local ethical committee and authorized by the national authority (Regierungspraesidium).

Apparatus. The crows sat on a wooden perch placed inside of an operant conditioning chamber in front of a touchscreen (3M Microtouch, 15 inch, $60 \mathrm{~Hz}$ refresh rate). Viewing distance to the monitor was $14 \mathrm{~cm}$. The program CORTEX (National Institute of Mental Health) presented the stimuli and stored behavioral data. An automated feeder delivered either mealworms (Tenebrio molitor larvae) or bird seed pellets upon correctly completed trials. During each trial, crows were trained to keep their head still in front of the computer display. This was controlled via a reflector foil attached to the crow's head. A trial only started when the crow moved its head into the beam of an infrared light barrier and kept its head still throughout the trial, thus ensuring stable head position. Whenever the crow made premature head movements and thereby left the infrared light barrier with its head during an ongoing trial, the computer terminated the trial and the trial was discarded.

Behavioral protocol. Crows discriminated numerosities in a delayed match-to-numerosity task (Fig. $1 A$ ): The crow started a trial by positioning its head in front of the monitor, thus closing an infrared light barrier, and maintaining the head still throughout the trial. As soon as the crow closed the infrared light barrier, an empty gray background circle was shown for $600 \mathrm{~ms}$ (presample phase), followed by a display showing the sample numerosity. The sample stimulus disappeared after $800 \mathrm{~ms}$ and the crow had to memorize the sample for $1000 \mathrm{~ms}$ during the delay phase, during which only the gray background circle was visible. In the following test phase, the test 1 display was a match in $50 \%$ of the cases; that is, it contained the same number of dots as the sample stimulus, but differed in appearance. The crow had to respond to indicate a numerical match by moving its head out of the light barrier. In the other $50 \%$ of the cases, test 1 was a non-match showing more or fewer dots than the sample display; here, the crow had to refrain from responding to the test 1 stimulus (i.e., keeping its head still within the light barrier) and wait for $800 \mathrm{~ms}$; afterward, the test2 display appeared, which always showed a match, and the bird had to respond by a head movement to indicate the match. Head 
movements before the test period aborted the trial automatically. Error trials led to a timeout of $3 \mathrm{~s}$. Correct trials were rewarded with food via the automated feeder.

Stimuli. The stimuli consisted of dot displays with varying numbers of dots (Fig. $1 B)$. Black dots $\left(0.4^{\circ}\right.$ to $2.5^{\circ}$ of visual angle) compromising a set were drawn on a gray background circle ( $12.3^{\circ}$ visual angle) shown in the center of the screen. Seven numerosities were used: 1, 2, 4, 7, 12, 20, and 30. Each of these numerosities served as sample and test stimulus. The sample and match numerosities within one trial were always indicated by different displays, thus preventing the crows from matching visual patterns. The displays were generated using a custom-written MatLab script. The dot arrangements were pseudorandomly generated and exchanged by new displays on a daily basis. Newly generated stimuli for each session prevented the crows from simply memorizing the visual patterns to solve the task. One stimulus batch contained 12 unique displays for each numerosity for each session (six standard and six control displays per numerosity). To further control for low-level visual features that may covary with changing numbers of dots, two stimulus sets were shown every session: The "standard" trial stimuli showed dots of pseudorandom size arranged randomly (but nonoverlapping) on the background circle. The "control" trial stimuli showed dots with both equal dot area and equal dot density combined across all numerosities. "Dot density" was defined as average distance between (the centers of) all dots on a numerosity display. "Dot area" was defined as cumulative surface area of all dots on a numerosity display; that is, the overall black area when individual black dots were added. Standard and control trials were randomly and unpredictably alternated.

Surgery and recordings. All surgeries were performed under sterile conditions while the animals were under general anesthesia. Crows were anesthetized with a ketamine/rompun mixture (50 mg of ketamine, $5 \mathrm{mg}$ of xylazine/kg body weight initially and supplemented by hourly $17 \mathrm{mg}$ of ketamine, $1.7 \mathrm{mg}$ of xylazine $/ \mathrm{kg}$ body weight, i.m.). After the surgery, the crows received analgesics (Butorphanol, trade name Morphasol, $1 \mathrm{mg} /$ $\mathrm{kg}$, i.m.). The head was placed in the stereotaxic holder that was customized for crows with the anterior fixation point (i.e., beak bar position) $45^{\circ}$ below the horizontal axis of the instrument. Using stereotaxic coordinates (center of craniotomy: AP $5 \mathrm{~mm}$; ML $13 \mathrm{~mm}$ ), we chronically implanted two microdrives with four electrodes each, a connector for the head stage and a small head post to hold the reflector for the light barrier. Glass-coated tungsten microelectrodes with $2 \mathrm{M} \Omega$ impedance (Alpha Omega) were used. The electrodes targeted the NCL. Tracing electrode tracks of an identically implanted crow used for a different study (Veit et al., 2014) confirmed that recording locations were within NCL. Cryostat sections were immunohistochemically stained for tyrosine hydroxylase to identify dopaminergic cells, which characterize the NCL (Veit and Nieder, 2013). Both crows used in this study are still alive and are participating in related experiments.

At the start of each recording session, the electrodes were advanced manually until a good neuronal signal was detected on at least one of the channels of each microdrive. Neurons were not preselected for involvement in the task. Each microdrive had a range of $\sim 6 \mathrm{~mm}$, which was exploited to record from the NCL across different depths over a period of several weeks. Signal amplification, filtering, and digitizing of spike waveforms were accomplished using the Plexon system.

For each recording session, the birds were placed in the recording setup and a head stage containing an amplifier was plugged into the connector implanted on the bird's head and connected to a second amplifier/filter and the Plexon MAP box outside of the setup by a cable above and behind the bird's head (all components by Plexon). Spike sorting into single-unit waveforms was performed manually offline using the Plexon system.

Data analysis. Behavioral performance (percentage correct) was calculated by counting all correctly performed trials and dividing this number by the number of all trials for each session. More precisely, responses to the test1 (match stimulus) in match trials and responses to the test2 (match stimulus) in non-match trials were correct. Details about the crows' behavioral performance can be found in Ditz and Nieder (2016).

The analysis included all neurons that were recorded for at least 4 repetitions of each sample numerosity per protocol type (average repe- tition number was 22) and had a firing rate of at least $0.5 \mathrm{~Hz}$ during all periods. Each recording session lasted between 350 and 710 correct trials in $\sim 2 \mathrm{~h}$.

Neuronal activity during the task was analyzed separately for the sample and delay phase. In the sample period, neuronal response rates were measured in a $800 \mathrm{~ms}$ window shifted by $100 \mathrm{~ms}$ from sample onset to account for the visual latency of most neurons (Veit et al., 2014). In the delay period, neuronal activity was analyzed in a $900 \mathrm{~ms}$ window shifted by $200 \mathrm{~ms}$ from sample offset. Because crow NCL neurons show an average visual response latency of $144 \mathrm{~ms}$ (Veit et al., 2014), the first 100 $\mathrm{ms}$ of the delay and test phase contain responses of the sample and delay period, respectively.

To determine the numerosity selectivity of the neurons, a two-factorial ANOVA was performed with numerosity (one to 30) and stimulation condition (standard or control) as factors. For each recording session, the highly variable standard protocol and the control protocol were used. Regardless of the control protocol, very few neurons responded to nonnumerical visual features of the stimulus displays, confirming that sensory parameters cannot account for the prominent numerosity effects. Only cells showing a significant main effect for numerosity $(p<0.01)$, but no significant main effect for stimulus type (standard vs control) or interaction were classified as "numerosity selective" and the numerosity eliciting the largest spike rate was defined as "preferred numerosity" of a given cell.

A cross-validation analysis was performed to estimate the reliability of preferred numerosity determination, also in terms of recording stability. The preferred numerosity of a cell was determined in the first half of the data (i.e., the first half of the recorded trials of a certain condition per neuron) and compared with the activity obtained in the second half of the data. To correlate these data, the preferred numerosity derived for the first half of the data was plotted against the preferred numerosity obtained for the second half of the data. This was done for the entire population of numerosity-selective neurons and the relationship between preferred numerosities in both datasets was quantified with the following simple regression technique:

$$
y=a+b * x
$$

where $y$ is the preferred numerosity of the neurons for the first half of the data, $x$ is the preferred numerosity of the neurons for the second half of the data, $a$ is the intercept, and $b$ is the slope of the $x-y$ relationship). If both datasets resulted in identical preferred numerosities, then the Pearson's linear correlation coefficient is 1 .

The activity rates were normalized by setting the maximum activity to the most preferred numerosity as $100 \%$ and the activity to the least preferred quantity as $0 \%$, thus creating neural filter functions. To evaluate the symmetry and the width of the numerosity tuning curves, Gaussian functions were fitted (MatLab Curve Fitting Toolbox) to these tuning functions plotted on four different scales: a linear scale, a power function with exponent of 0.5 , a power function with exponent of 0.33 , and a logarithmic $\left(\log _{2}\right)$ scale. The scales were chosen based on the theories of Fechner (1860) and Stevens (1961). Fechner suggested that the relationship between a sensory stimulus and its sensation is of logarithmic nature, whereas Stevens claimed that the relationship is based on a power law. The more symmetrical the plotted tuning curves appeared on a given scale, the better the resulting fit $\left(r^{2}\right)$ with the Gaussian functions. The better the fit, the better the given scale describes the relationship between the physical magnitude and its neuronal representation. The widths of the Gaussian fits $(\sigma)$ were evaluated to test for the neuronal magnitude effect. If a magnitude effect is present, the sigmas of the linear Gaussian fits should increase when plotted against numerosity; the sigmas should be stable if the scaling is perfectly counteracting the proportional broadening of the performance functions.

To evaluate the behavioral significance of numerosity-selective neurons, discharges in correct and error trials were compared. Of all purely numerosity-selective neurons, neurons with at least three error trials for their preferred numerosity were included in analyses of error trials. Discharge rates of single neurons to the preferred numerosity were compared in correct versus error trials (Wilcoxon test). 

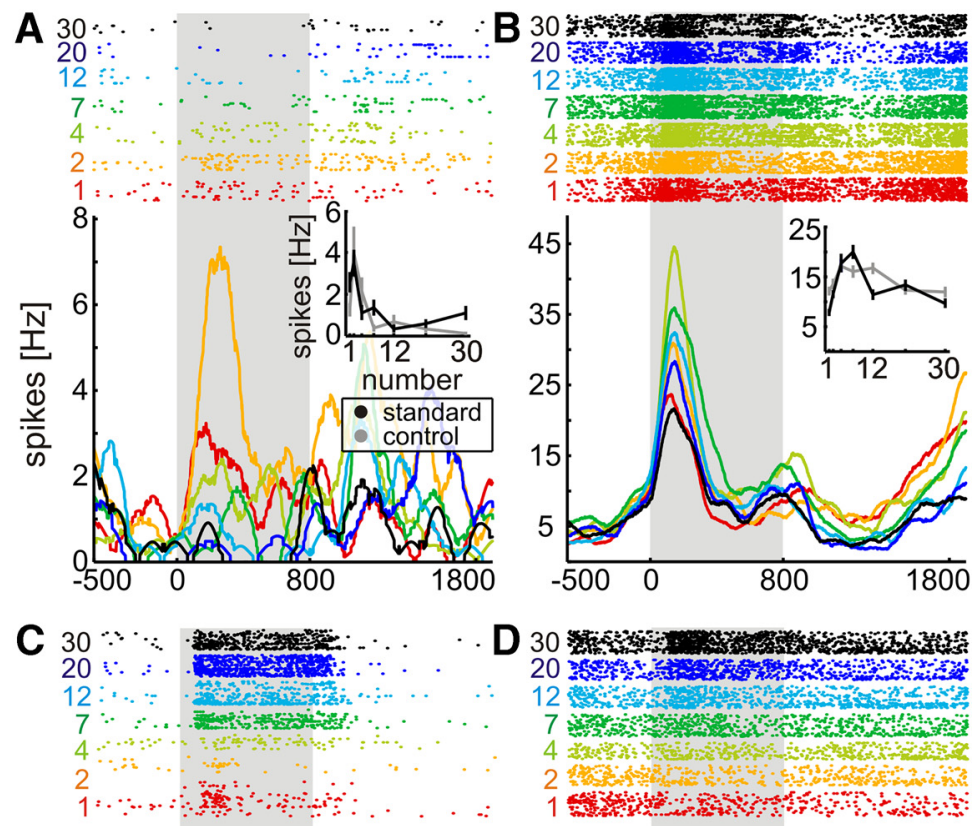

\section{20
12
7
4
2
1}
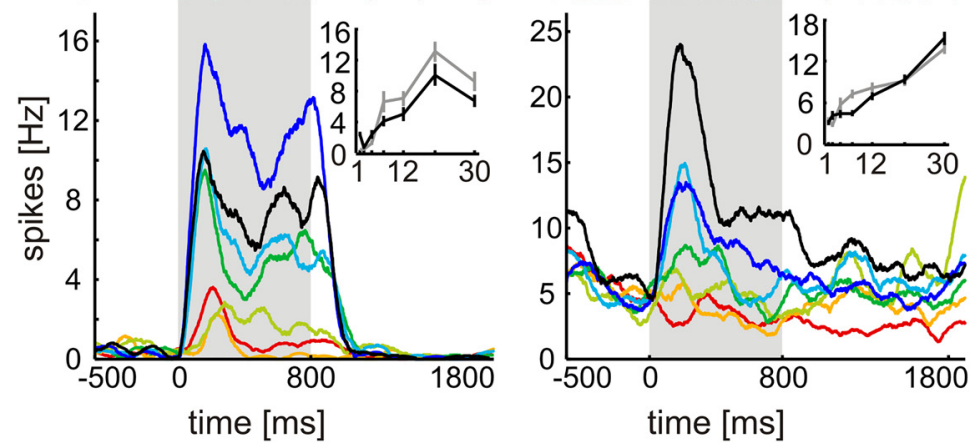

Figure 2. Responses of four example NCL neurons selective to numerosity $2(\boldsymbol{A}), 7(\boldsymbol{B}), 20(\boldsymbol{C})$, and $30(\boldsymbol{D})$ during the sample period. Top, Dot-raster histograms with each dot representing one action potential. Bottom, Averaged spike density function (activity averaged and smoothed by a $150 \mathrm{~ms}$ Gaussian kernel). Colors in the dot-raster and spike density functions correspond to the shown quantity during the sample period (gray shading). Inset, Tuning function with the mean activity in standard (black line) and control trials (gray line) of the neurons to numerosity in the sample period. Error bar indicates \pm SEM.

better than chance (50\%) for all sample numerosities relative to the numerically most distant non-matches (binomial test, $p<$ $0.01)$. Even though both crows showed a behavioral bias toward match trials, behavioral performance was significant for both match and non-match conditions separately, as well as for their combined performance. A detailed analysis of the crows' performance can be found in Ditz and Nieder (2016). The crows readily generalized to the control stimulus sets; performance was very similar across them. This suggests that crows were indeed judging numerosity. The crows' discrimination was based on an analog magnitude system and showed the Weber-fraction signature; that is, the "just noticeable difference" between numerosity pairs increased in proportion to the numerical magnitudes. The detailed analysis of the performance indicates that numerosity representations in crows are scaled on a logarithmically compressed "number line."

\section{Neuronal sample activity}

We recorded from 325 randomly selected neurons from the NCL of the two crows while they performed the numerosity discrimination task. Of these cells, 22\% (72/ 325) modulated their discharges as a function of numerosity during sample presentation. This selectivity was found regardless of the exact appearance of the multipledot patterns. Only cells showing a significant numerosity effect, but no significant effect of stimulus type (standard vs control) or interaction, were classified as numerosity-selective neurons according to a two-factor ANOVA $(p<0.01)$.

Four such neurons that generalized

\section{Results}

\section{Behavior}

Two crows performed a delayed matching-to-sample task with numerosity as discriminanda. The crows matched the number of dots $(1,2,4,7,12,20$, and 30 dots $)$ presented on touch-sensitive computer displays (Fig. $1 A, B$ ). Crows watched 2 displays (first sample, then test) separated by a $1 \mathrm{~s}$ delay. They were trained to move their head to leave the infrared light barrier if the test displays contained the same number of items as the sample.

To exclude that the crows used covarying visual parameters rather than numerical quantity to solve the task, sample and test displays were never identical and all displays were generated and exchanged before every session. The exact physical appearance of the many different numerosity displays was varied randomly. In addition to a standard protocol with pseudorandomly chosen dot sizes and arbitrary dot locations, we also used a control protocol in which the total area of the dots and the average density of the dots were kept constant across all numerosities (Fig. 1B). Therefore, across these stimulus sets, the exact physical appearance of each numerical quantity varied widely.

Both crows performed the task proficiently $(79.5 \pm 0.06 \%$ and $78.9 \pm 0.07 \%$ correct over all recording sessions for crow A and crow $\mathrm{J}$, respectively). Average performance of both crows was significantly across changes in the physical appearance of the sample displays (according to the ANOVA) are shown in Figure 2. The example neuron shown in Figure $2 A$ was tuned to numerosity 2 and showed indifferent activity to the standard versus control protocol. Other neurons were tuned to numerosity 7 (Fig. 2B), numerosity 20 (Fig. $2 C$ ), or numerosity 30 (Fig. $2 D$ ), again responding equally well to standard and control protocols.

Each neuron showed peak activity for one of the numerosities and a systematic drop-off of activity as the number of sample items varied from the preferred value. Neural preference was distributed across all seven displayed numerosities (numerosity 1: 19\%; 2: 21\%; 4: 6\%; 7: 7\%; 12: 17\%; 20: 12\%; 30: 18\%; Fig. 3A). Few cells (not included in the group of numerosity-selective neurons) were responsive to both numerosity and protocol type (9\% or 28/325; 2-way ANOVA, effect of numerosity and stimulus protocol or interaction between stimulus protocol and numerosity, $p<0.01)$. Therefore, the numerosity of sample items was the dominant factor encoded by these neurons, not the physical appearance of the displays.

To estimate the reliability of preferred numerosity determination, a cross-validation analysis (see Materials and Methods) was performed. A correlation coefficient of 1 would indicate a perfect match between the preferred numerosity in the first half of the 

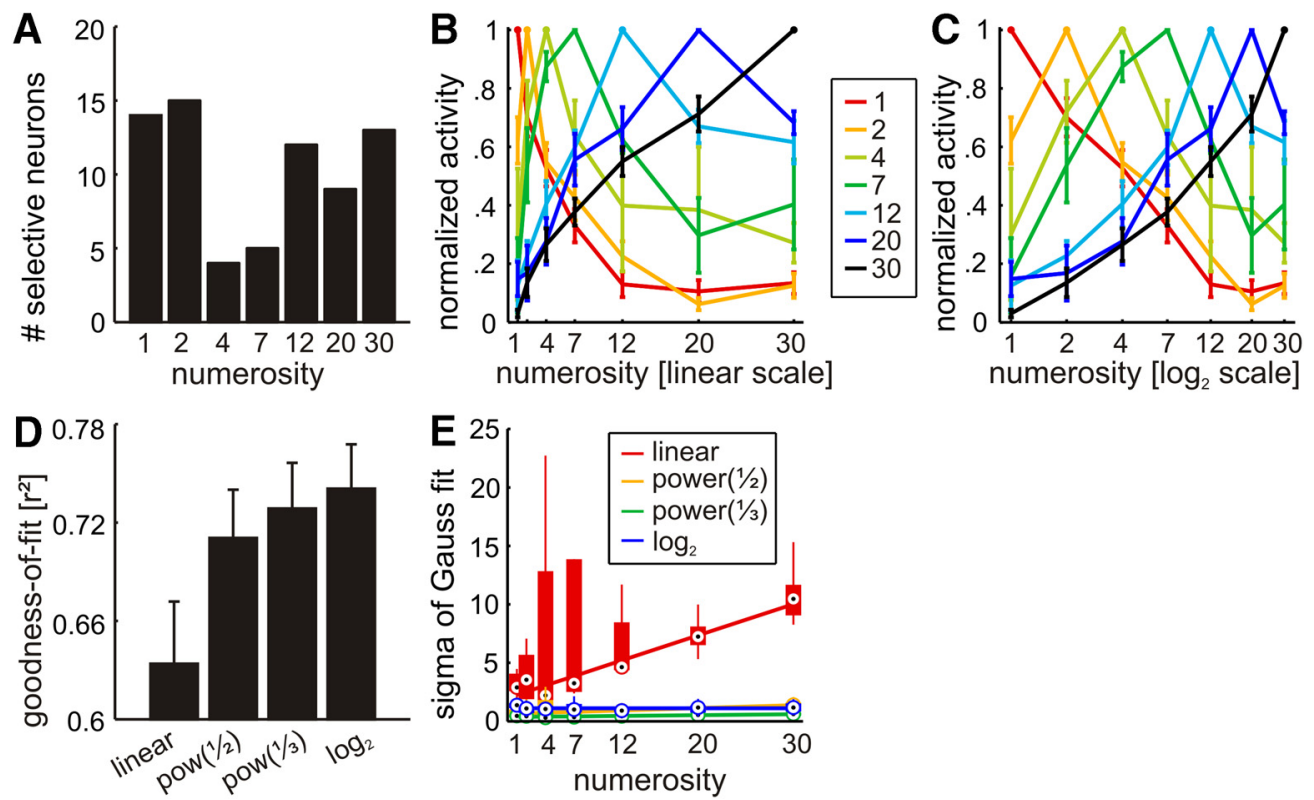

Figure 3. Neuronal numerosity tuning during the sample period. $\boldsymbol{A}$, Frequencies of numerosity-selective neurons specified for their preferred numerosity during the sample period. $\boldsymbol{B}, \boldsymbol{C}$ Normalized tuning functions averaged for neurons preferring the same numerosity (indicated by same color) when plotted on a linear scale ( $\boldsymbol{B}$ ) or logarithmic number scale ( $\boldsymbol{C}$ ). Error bar indicates \pm SEM. $\boldsymbol{D}$, Averaged goodness-of-fit $\left(r^{2}\right)$ to the filter functions on the four different scaling schemes. Error bar indicates \pm SEM. $\boldsymbol{E}$, Widths $(\sigma)$ of fitted Gaussian functions of performance curves as a function of sample numerosity and scaling scheme. The colors indicate the scaling scheme, the edges of the boxes the 25th and 75 th percentile, and the black dot inside the boxes the respective median. The colored lines indicate linear fits to the medians.

trials with the numerosity in the second half of the trials for each neuron. The calculated correlation coefficient of $r=0.83$ ( $p<$ $0.0001, n=72$ ) indicates a highly reproducible preferred numerosity for the sample phase. The neurons' preferred numerosity was reliable and robust.

Neurons showed peak activity to a specific number of items and a progressive drop-off as the numerosity progressively varied, resulting in a systematic, orderly representation of numerosity (Fig. $2 A-D$ ). This was evaluated across the population. Population neural tuning functions were calculated by averaging the normalized activity for all neurons that preferred a given quantity. Neural activity formed band-pass filters with increasingly attenuated activity as distance from the preferred quantity increased (Fig. 3B). The neuronal data mirrored the behavioral distance and magnitude effects by the fact that the neural filters were also peak functions that became less selective (wider) with increasing preferred numerosity.

\section{Scaling of sample tuning functions}

We investigated the coding scheme by plotting the tuning functions on different number scales. When plotted on a linear number scale, the shapes of the tuning functions were asymmetric with a steeper slope toward smaller numerosities (Fig. 3B). However, when the same tuning functions were plotted on a logarithmic axis, the shapes were approximately Gaussian, suggesting a logarithmic representation of numerosities (Fig. $3 C$ ). The same nonlinear compression effect was also observed for the behavioral performance functions (Ditz and Nieder, 2016). We verified this finding by fitting Gauss functions to the tuning functions when plotted on a linear or three nonlinear scales with increasing compression, namely power functions with exponent 0.5 and 0.33 or a logarithmic scale. The goodness-of-fit $\left(r^{2}\right)$ values of the Gauss fits, which were taken as a quantitative measure of the tuning curves' symmetry, differed between the four scaling schemes $(p<0.0001$, Friedman test, $n=72$; Fig. 3D).
The mean goodness-of-fit values for the linear scale, the power function with exponent of 0.5 , the power function with exponent of 0.33 , and the logarithmic scale were $0.63,0.71,0.73$, and 0.74 , respectively. The logarithmic scale provided a better fit to the data than the linear scale $(p<0.0034$, Wilcoxon test, $n=$ 72 ). No difference in the goodness-of-fit values were found between the three nonlinear (the two power functions and the logarithmic) scales $(p>0.05$, Wilcoxon test, $n=72)$. Similar results were found for the behavioral performance functions (Ditz and Nieder, 2016). As predicted by a nonlinear coding model (Nieder and Miller, 2003), the widths ( $\sigma$ derived by Gauss fits) of the tuning functions were nearly constant with increasing preferred numerosities when the data were plotted on a logarithmic scale (slope of linear fit $=-0.0004$ ), but increased with numerosity when the data were plotted on a linear scale (slope $=0.2676$; Fig. $3 E)$. In terms of the scaling scheme, the neural data mirrored behavioral findings (Ditz and Nieder, 2016).

\section{Neuronal delay activity}

The delayed match to sample task requires maintenance of numerosities in working memory during the delay period. We next determined the selectivity of NCL neurons to the remembered numerosities. Of the 325 cells recorded, 21\% (68/325) could be classified as being numerosity selective and only showed a significant numerosity effect, but no significant effect of stimulus type (standard vs control) or interaction (two-factor ANOVA) $(p<$ $0.01)$. A further $8 \%(25 / 325$; not included in the group of numerosity-selective neurons) were responsive to both numerosity and stimulus type (two-way ANOVA, effect of numerosity and stimulus protocol or interaction between stimulus protocol and numerosity, $p<0.01)$. Examples of delay-selective numerosity cells are shown in Figure 4. Neurons showed ramping activity toward the end of the delay period selective for numerosity 1 (Fig. 4A), numerosity 2 (Fig. 4B), numerosity 12 (Fig. 4C), and numerosity 20 (Fig. 4D). Again, numerosities closer to the pre- 
A $30 \ldots$

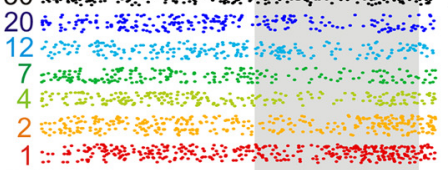
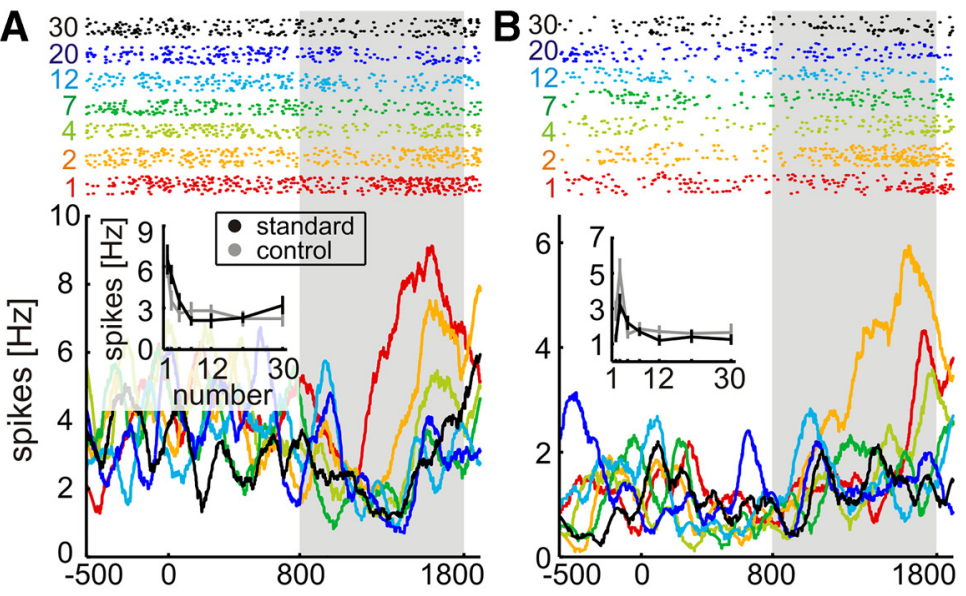

C $300 \%$ H

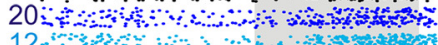
12 a

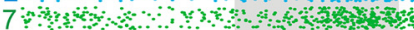
4 20 1 1.

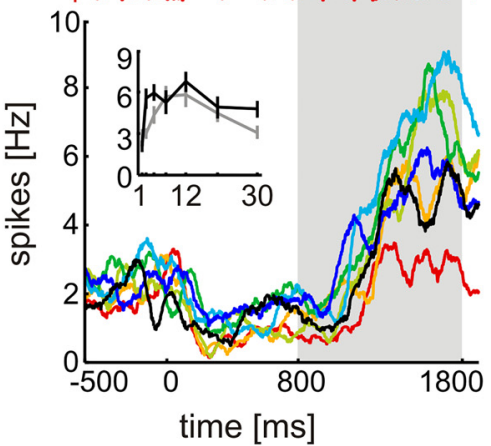

30 -

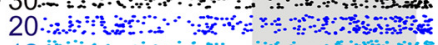

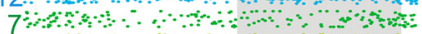
$40+30$ 2 1 a

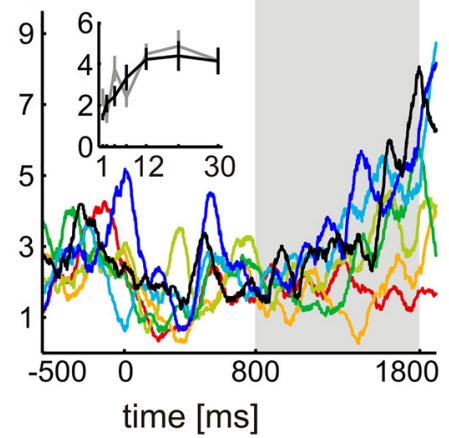
120 and

Figure 4. Responses of four example NCL neurons selective to numerosity $1(\boldsymbol{A}), 2(\boldsymbol{B}), 12(\boldsymbol{C})$, and $20(\boldsymbol{D})$ during the delay period (gray shading). Top, Dot-raster histograms with each dot representing one action potential. Bottom, Averaged spike density function (activity averaged and smoothed by a 150 ms Gauss kernel). Colors in the dot-raster and spike density functions correspond to the shown quantity during the sample period. Inset, tuning function with the mean activity in standard (black line) and control trials (gray line) of the neurons to numerosity in the delay period. Error bar indicates \pm SEM.

ferred numerosity elicited more similar discharge rates, whereas remote numerosities showed close to baseline activity, thus giving rise to peak tuning curves. Of the 72 numerosity-selective cells during the sample phase, 25 cells were also numerosity selective during the delay period. On average, the neurons' preferred numerosity during the delay was comparable to the same neurons' preferred numerosity during the sample phase $(r=0.473 ; p=$ $0.017 ; n=25)$. For those 25 neurons tuned in both the sample and delay period, delay activity robustly persisted throughout the delay period and showed "ramping" activity toward the end of the delay phase.

The neurons' preferred numerosity was robust throughout the recording. A correlation coefficient of $r=0.78(p<0.0001$, $n=68$ ) indicated a stable and reproducible preferred numerosity between the first and second half of the trials for each neuron during the delay phase. Neural preference was distributed across all seven displayed numerosities (numerosity 1: 22\%; 2: 25\%; 4 : 3\%; 7: 6\%; 12: 9\%; 20: 18\%; 30: 18\%; Fig. $5 A$ ). Just as in the sample phase, population neural tuning functions in the delay phase (Fig. 5B) showed a neuronal correlate of the distance effect (peak functions) and the magnitude effect (broader tuning with increasing preferred numerosity).

\section{Scaling of delay tuning functions}

We again investigated the best representational scheme by plotting the delayselective tuning functions on the four differently scaled number axes. The shapes of the tuning functions were asymmetric when plotted on a linear scale (Fig. $5 B)$, but symmetric when plotted on a logarithmic axis (Fig. 5C). The goodness-offit $\left(r^{2}\right)$ values of the Gaussian fits differed between the four scaling schemes $(p<$ 0.001, Friedman test, $n=68$; Fig. $5 D$ ). The mean goodness-of-fit values for the linear scale, the power function with exponent of 0.5 , the power function with exponent of 0.33 , and the logarithmic scale were $0.70,0.75,0.76$, and 0.77 , respectively. The (nonlinear) logarithmic scale provided a better fit to the data than the linear scale $(p<0.0048$, Wilcoxon test, $n=68$ ). No difference in the goodness-offit values were found for between the three nonlinear (the two power functions and the logarithmic) scales $(p>0.05$, Wilcoxon test, $n=68$ ). The widths ( $\sigma$ derived by Gauss fits) of the tuning functions were more or less constant with increasing preferred numerosities when the data were plotted on a logarithmic scale (slope of linear fit $=-0.0047)$, but increased with numerosity when the data were plotted on a linear scale (slope $=0.2769$; Fig. $5 E$ ). Again, this was reminiscent of the effects found for the crows' behavioral discriminations.

\section{Comparison of discharges during correct and error trials}

If the responses of numerosity-selective neurons are directly related to the crows' number judgments, then numerosity tuning should be degraded in trials in which crows made erroneous decisions. Indeed, neural activity for the preferred numerosity was significantly reduced to $76 \%$ in the sample period $(p<0.01$, Wilcoxon test, $n=68)$ and $69 \%$ in the delay period $(p<0.05$, Wilcoxon test, $n=64$ ) of that observed on correct trials (normalized to $100 \%$ ). This finding is illustrated by a "clipping" of the averaged tuning peak and by enhanced discharges to nonpreferred numerosities on error trials (Fig. 6). This change in activity may reflect the sample being mistakenly encoded and/or memorized as an adjacent numerosity. This suggests that the crows tended to make judgment errors whenever the neurons were not able to encode their preferred numerosity properly.

\section{Discussion}

We report a neuronal correlate for sensory representation and working memory for a broad range of numerosities in the crow NCL, a higher association brain area of birds. Numerosityselective NCL neurons showed clear peaked tuning functions, suggesting a pure labeled-line code for numerosity judgments. Just as the behavioral performance functions, the neuronal tuning functions obeyed the Weber-Fechner Law and were best represented on a logarithmically compressed number line. Com- 

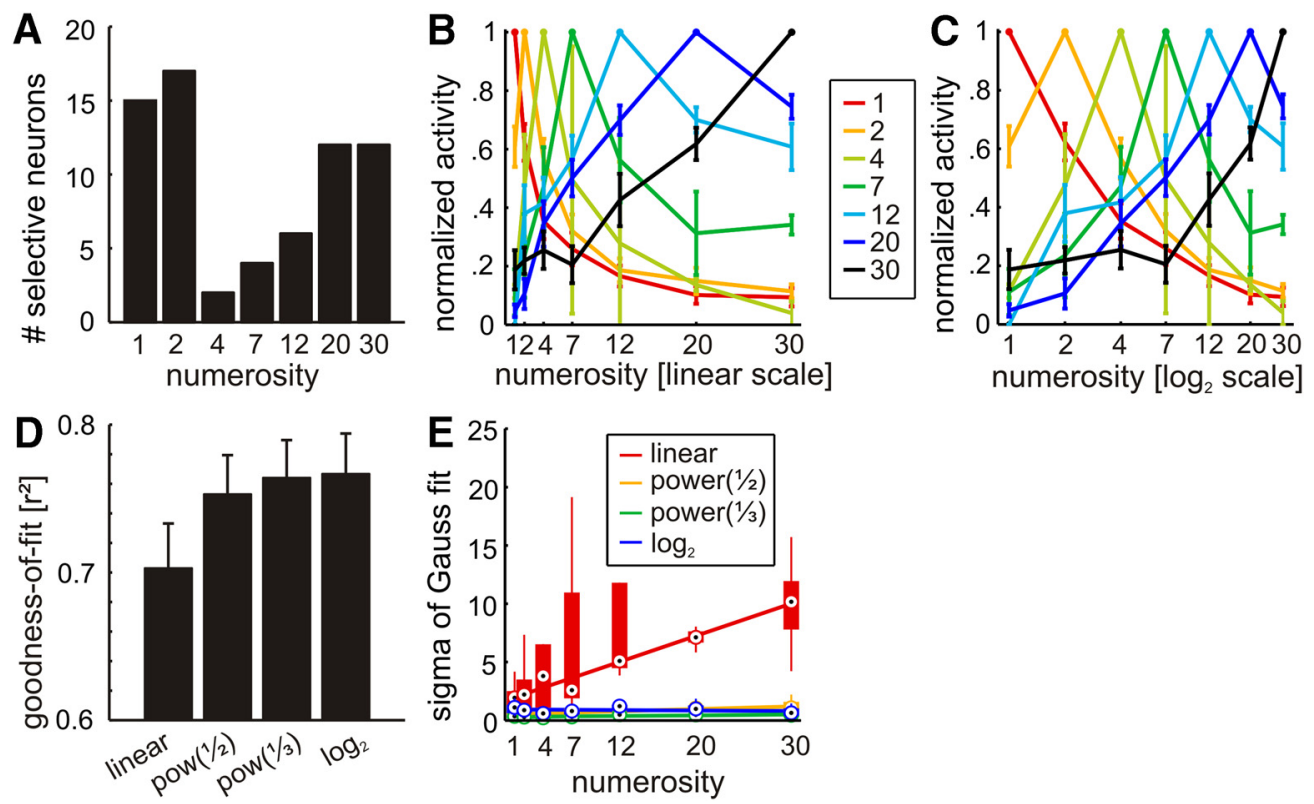

Figure 5. Neuronal numerosity tuning during the delay period. $\boldsymbol{A}$, Frequencies of numerosity-selective neurons specified for their preferred numerosity during the delay period. $\boldsymbol{B}, \boldsymbol{C}$, Normalized tuning functions averaged for neurons preferring the same numerosity (indicated by same color) when plotted on a linear $(\boldsymbol{B})$ or logarithmic $(\boldsymbol{C})$ number scale. Error bar indicates \pm SEM. $\boldsymbol{D}$, Averaged goodness-of-fit $\left(r^{2}\right)$ to the filter functions on the four different scaling schemes. Error bar indicates \pm SEM. $\boldsymbol{E}$, Widths $(\sigma)$ of fitted Gaussian functions of performance curves as a function of sample numerosity and scaling scheme. The colors indicate the scaling scheme, the edges of the boxes the 25 th and 75 th percentile, and the black dot inside the boxes the respective median. The colored lines indicate linear fits to the medians.
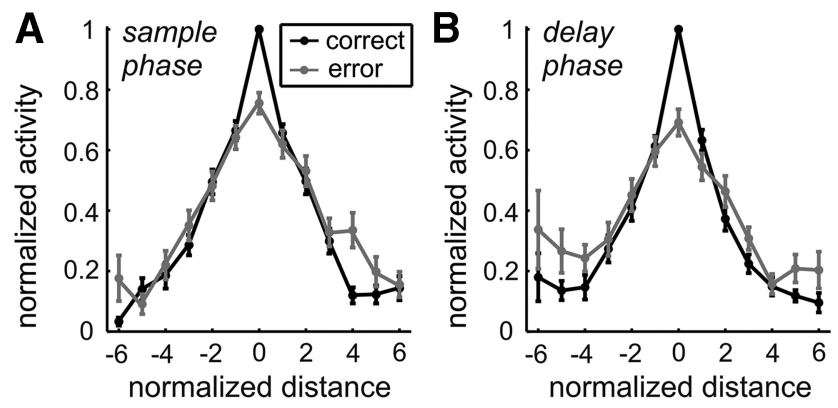

Figure 6. Behavioral relevance of numerosity-selective neurons. Normalized average tuning function across all neurons that are numerosity selective during the sample $(\boldsymbol{A} ; n=68)$ and delay ( $\boldsymbol{B} ; n=64)$ periods. Functions for correct (black lines) and error trials (gray lines) are shown. Error bar indicates \pm SEM. The distances between the tested numerosities are treated as having the distance 1 .

parison of NCL data with recordings in primates suggests a common code for number in at least two different vertebrate taxa.

\section{Analog magnitude system}

Two main mechanisms to represent the number of items are discussed. An object tracking system for small numbers (also termed "subitizing") is thought to allow precise number representations, but only for up to four items (set-size limit; Feigenson et al., 2004). Such a set-size limit for numerosities was absent in crows (Ditz and Nieder, 2016). Instead, numerosities much larger than four were represented both behaviorally and neuronally, clearly demonstrating the presence of an analog magnitude system. The analog magnitude system can represent an unlimited amount of set sizes and shows all characteristics postulated by the Weber-Fechner law, most importantly the ratio-dependent discrimination, both for small and large numerosities. The systematic increase of the widths of the tuning functions with increasing stimulus magnitude attests a neural foundation for the ratio- dependent number discriminations. This was previously observed for small numerosities from one to five (Ditz and Nieder, 2015) and now also demonstrated for large numerosities. Both the behavioral and the neuronal data can be explained by the analog magnitude system only. This is in agreement with recordings from macaque monkeys (Nieder and Miller, 2004; Nieder and Merten, 2007).

\section{Labeled-line code}

Neurons in the corvid NCL were tuned to individual preferred numerosities. Cells were found that encoded each of the tested sample numerosities from one to 30 as preferred numerosity. This is characteristic of a labeled-line code by which each numerosity can be unequivocally encoded via discharges of the neuronal population. Such a labeled-line code for explicit numerosity representations was present for small (Ditz and Nieder, 2015) and, as demonstrated in the current study, also for large numerosities in the corvid NCL.

As predicted by the Weber-Fechner law for sensory magnitudes, the neuronal tuning curves to large numerosities were best described - that is, more symmetric - on a logarithmic number scale. This logarithmic coding scheme with symmetric bellshaped tuning curves has the advantage of providing a set of neurons with the same widths of tuning for all numerosities. Moreover, this scaling scheme constitutes the variability of neuronal responses independently of number preference.

The logarithmic scaling at the neuronal level is in agreement with the same scaling scheme at the behavioral level in crows (Ditz and Nieder, 2016), suggesting that NCL number neurons are the basis of the crows' number discrimination capability. Further evidence that the neuronal discharges are behaviorally relevant stems from the analysis of error trials. In error trials, responses to the preferred numerosity were significantly reduced. Therefore, whenever the neurons failed to properly encode their preferred numerosity, the crow was at risk to err. Similar error 
trial effects have been reported repeatedly for numerosityselective neurons in monkeys (Nieder et al., 2002; Nieder and Merten, 2007).

Our data from the crow NCL can be compared directly with cortical data in nonhuman primates; similar task protocols and analyses of neuronal data have been used widely to investigate the neuronal number code in behaving monkeys. The numerosity code found in the crow endbrain is surprisingly reminiscent of findings for neurons in the monkey PFC and posterior parietal cortex (PPC) (Nieder, 2013). In monkeys that discriminated visual numerosity in dot arrays (Eiselt and Nieder, 2013; Jacob and Nieder, 2014; Ott et al., 2014; Viswanathan and Nieder, 2015; Ott and Nieder, 2016; Ramirez-Cardenas et al., 2016; Nieder, 2016b), enumerated numerosity sequentially one by one in visual displays (Nieder et al., 2006), or performed hand movements (Sawamura et al., 2002, 2010), tuned numerosity detectors were found. In the macaque PFC, neurons were also tuned supramodally to numerosity; that is, regardless of whether visual dots or acoustic sounds had to be assessed across time (Nieder, 2012). Finally, neurons in monkeys performing a sequence of hand movements were also tuned to preferred numbers of movements (Sawamura et al., 2002; Sawamura et al., 2010). Even if monkeys were numerically naive and unaware of numerosities, a fraction of the neurons in the PFC and IPS were tuned to numerosities (Viswanathan and Nieder, 2013).

Our single-cell data in the corvid brain are not only in agreement with nonhuman primate studies (Nieder, 2013), but also concur with functional imaging studies in humans. fMRI adaptation indirectly revealed peaked tuning profiles in BOLD signals in the human IPS (Piazza et al., 2004) and PFC (Jacob and Nieder, 2009). Similar to our findings in crows and monkeys, these BOLD tuning curves also showed ratio-dependent tuning widths and are best described on a logarithmic number scale. This commonality suggests that songbirds, nonhuman primates, and humans share a comparable labeled-line coding mechanism for the representation of a broad range of numerosities that obeys the Weber-Fechner Law.

\section{Working memory}

The delayed match-to-sample task required crows to retain numerosity information briefly in working memory to subserve a future comparison and choice process during the test period. Previous lesion studies in pigeons have shown that NCL is causally involved in mastering delayed response tasks (Mogensen and Divac, 1993; Diekamp et al., 2002). A key neurophysiological signature of working memory is sustained (or persistent) activity after the stimulus has ceased (Veit et al., 2014). As a putative correlate of working memory for numerosity, a significant proportion $(21 \%)$ of NCL neurons showed sustained activity during the delay period. Similar to numerosity-selective neurons in the sample phase, delay-selective NCL neurons were tuned to preferred numerosity. All effects found for sample-selective number neurons, the labeled-line code as well as logarithmic scaling, equally applied to delay-selective neurons. Moreover, the responses to the preferred numerosity during error trials were even more decreased than during the sample period. This suggests that neuronal activity in the delay period is more closely correlated with the animals' numerical discriminatory behavior (Veit et al., 2014). Sustained and tuned activity of NCL neurons thus seems to be the tool to process relevant numerical information across time to use this information for goal-directed behavior about numerical quantities.

Similar to the avian NCL, the primate PFC is thought to be a key area involved in working memory (Fuster and Alexander, 1971; Miller et al., 1996; Rainer and Miller, 2002). Working memory representations for numerical quantity have been reported in the PPC and PFC of monkeys performing a very similar match-to-numerosity task (Nieder, 2013). Across many studies, a significant proportion of neurons $(20-30 \%$ in the PFC and $10-$ $20 \%$ in the PPC) show persistent activity during delay periods when monkeys memorize the numerosity that they have just sensed (Nieder, 2016a). In addition, many delay-selective neurons not only signal the number of items in dot patterns, but also integrate across spatial and temporal presentation formats and visual-auditory modalities (Nieder et al., 2006; Nieder, 2012). This suggests that the primate PFC and the corvid NCL share a similar function in storing numerical information in working memory.

\section{Comparative neuroscience of numerical cognition}

We show that the labeled-line code for the analog number system is not only found in the primate neocortex, but also in the avian endbrain. This is surprising given that crows and primates have very dissimilar organized endbrain structures, yet both endbrains independently evolved a very similar mode of coding numerical information based on convergent evolution. This suggests that this code may be computationally superior to alternative representations such as summation coding, in which numerosity is encoded via monotonic response functions of neurons (Roitman et al., 2007). We think the realm of numerical cognition is particularly suited to tackle coding principles for abstract information, not only in the mammalian brain, but in the vertebrate brain in general (Clayton and Emery, 2015). Ultimately, only a comparative approach will help to decipher evolutionary stable neuronal mechanisms and codes (Bullock, 1984).

\section{References}

Bogale BA, Kamata N, Mioko K, Sugita S (2011) Quantity discrimination in jungle crows, Corvus macrorhynchos. Animal Behavior 82:635-641. CrossRef

Bullock TH (1984) Comparative neuroscience holds promise for quiet revolutions. Science 225:473-478. CrossRef Medline

Butler AB, Reiner A, Karten HJ (2011) Evolution of the amniote pallium and the origins of mammalian neocortex. Ann N Y Acad Sci 1225:14-27. CrossRef Medline

Clayton NS, Emery NJ (2015) Avian models for human cognitive neuroscience: a proposal. Neuron 86:1330-1342. CrossRef Medline

Diekamp B, Gagliardo A, Güntürkün O (2002) Nonspatial and subdivisionspecific working memory deficits after selective lesions of the avian prefrontal cortex. J Neurosci 22:9573-9580. Medline

Ditz HM, Nieder A (2015) Neurons selective to the number of visual items in the corvid songbird endbrain. Proc Natl Acad Sci U S A 112:78277832. CrossRef Medline

Ditz HM, Nieder A (2016) Numerosity representations in crows obey the Weber-Fechner law. Proc Biol Sci 283:20160083. CrossRef Medline

Divac I, Mogensen J, Björklund A (1985) The prefrontal "cortex" in the pigeon: biochemical evidence. Brain Res 332:365-368. CrossRef Medline

Eiselt AK, Nieder A (2013) Representation of abstract quantitative rules applied to spatial and numerical magnitudes in primate prefrontal cortex. J Neurosci 33:7526-7534. CrossRef Medline

Fechner GT (1860) Elemente der Psychophysik, Vol 3. Leipzig: Breitkopf \& Härtel.

Feigenson L, Dehaene S, Spelke E (2004) Core systems of number. Trends Cogn Sci 8:307-314. CrossRef Medline

Fuster JM, Alexander GE (1971) Neuron activity related to short-term memory. Science 173:652-654. CrossRef Medline

Güntürkün O (2005) The avian "prefrontal cortex" and cognition. Curr Opin Neurobiol 15:686-693. CrossRef Medline

Hoffmann A, Rüttler V, Nieder A (2011) Ontogeny of object permanence and object tracking in the carrion crow, Corvus corone. Animal Behavior 82:359-367. CrossRef 
Jacob SN, Nieder A (2009) Tuning to non-symbolic proportions in the human frontoparietal cortex. Eur J Neurosci 30:1432-1442. CrossRef Medline

Jacob SN, Nieder A (2014) Complementary roles for primate frontal and parietal cortex in guarding working memory from distractor stimuli. Neuron 83:226-237. CrossRef Medline

Jarvis ED, et al. (2005) Avian brains and a new understanding of vertebrate brain evolution. Nat Rev Neurosci 6:151-159. CrossRef Medline

Meck WH, Church RM (1983) A mode control model of counting and timing processes. J Exp Psychol Anim Behav Process 9:320-334. CrossRef Medline

Merten K, Nieder A (2009) Compressed scaling of abstract numerosity representations in adult humans and monkeys. J Cogn Neurosci 21:333-346. CrossRef Medline

Miller EK, Erickson CA, Desimone R (1996) Neural mechanisms of visual working memory in prefrontal cortex of the macaque. J Neurosci 16: 5154-5167. Medline

Mogensen J, Divac I (1993) Behavioural effects of ablation of the pigeonequivalent of the mammalian prefrontal cortex. Behav Brain Res 55:101107. CrossRef Medline

Moll FW, Nieder A (2014) The long and the short of it: rule-based relative length discrimination in carrion crows, Corvus corone. Behav Processes 107:142-149. CrossRef Medline

Moll FW, Nieder A (2015) Cross-modal associative mnemonic signals in crow endbrain neurons. Curr Biol 25:2196-2201. CrossRef Medline

Nieder A (2012) Supramodal numerosity selectivity of neurons in primate prefrontal and posterior parietal cortices. Proc Natl Acad Sci U S A 109: 11860-11865. CrossRef Medline

Nieder A (2013) Coding of abstract quantity by "number neurons" of the primate brain. J Comp Physiol A Neuroethol Sens Neural Behav Physiol 199:1-16. CrossRef Medline

Nieder A (2016a) The neuronal code for number. Nat Rev Neurosci 17: 366-382. CrossRef Medline

Nieder A (2016b) Representing something out of nothing: The dawning of zero. Trends Cogn Sci 20:830-842. CrossRef

Nieder A, Merten K (2007) A labeled-line code for small and large numerosities in the monkey prefrontal cortex. J Neurosci 27:5986-5993. CrossRef Medline

Nieder A, Miller EK (2003) Coding of cognitive magnitude: Compressed scaling of numerical information in the primate prefrontal cortex. Neuron 37:149-157. CrossRef Medline

Nieder A, Miller EK (2004) A parieto-frontal network for visual numerical information in the monkey. Proc Natl Acad Sci U S A 101:7457-7462. CrossRef Medline

Nieder A, Freedman DJ, Miller EK (2002) Representation of the quantity of visual items in the primate prefrontal cortex. Science 297:1708-1711. CrossRef Medline

Nieder A, Diester I, Tudusciuc O (2006) Temporal and spatial enumeration processes in the primate parietal cortex. Science 313:1431-1435. CrossRef Medline
Ott T, Nieder A (2016) Dopamine D2 receptors enhance population dynamics in primate prefrontal working memory circuits. Cereb Cortex. In press.

Ott T, Jacob SN, Nieder A (2014) Dopamine receptors differentially enhance rule coding in primate prefrontal cortex neurons. Neuron 84:13171328. CrossRef Medline

Piazza M, Izard V, Pinel P, Le Bihan D, Dehaene S (2004) Tuning curves for approximate numerosity in the human intraparietal sulcus. Neuron 44 547-555. CrossRef Medline

Rainer G, Miller EK (2002) Timecourse of object-related neural activity in the primate prefrontal cortex during a short-term memory task. Eur J Neurosci 15:1244-1254. CrossRef Medline

Ramirez-Cardenas A, Moskaleva M, Nieder A (2016) Neuronal representation of numerosity zero in the primate parieto-frontal number network. Curr Biol 26:1285-1294. CrossRef Medline

Roitman JD, Brannon EM, Platt ML (2007) Monotonic coding of numerosity in macaque lateral intraparietal area. PLoS Biol 5:e208. CrossRef Medline

Romo R, Brody CD, Hernández A, Lemus L (1999) Neuronal correlates of parametric working memory in the prefrontal cortex. Nature 399:470 473. CrossRef Medline

Sawamura H, Shima K, Tanji J (2002) Numerical representation for action in the parietal cortex of the monkey. Nature 415:918-922. CrossRef Medline

Sawamura H, Shima K, Tanji J (2010) Deficits in action selection based on numerical information after inactivation of the posterior parietal cortex in monkeys. J Neurophysiol 104:902-910. CrossRef Medline

Scarf D, Hayne H, Colombo M (2011) Pigeons on par with primates in numerical competence. Science 334:1664. CrossRef Medline

Stevens SS (1961) To honor Fechner and repeal his law: A power function, not a $\log$ function, describes the operating characteristic of a sensory system. Science 133:80-86. CrossRef Medline

Veit L, Nieder A (2013) Abstract rule neurons in the endbrain support intelligent behaviour in corvid songbirds. Nat Commun 4:2878. CrossRef Medline

Veit L, Hartmann K, Nieder A (2014) Neuronal correlates of visual working memory in the corvid endbrain. J Neurosci 34:7778-7786. CrossRef Medline

Veit L, Pidpruzhnykova G, Nieder A (2015a) Associative learning rapidly establishes neuronal representations of upcoming behavioral choices in crows. Proc Natl Acad Sci U S A 112:15208-15213. CrossRef Medline

Veit L, Hartmann K, Nieder A (2015b) Spatially tuned neurons in corvid nidopallium caudolaterale signal target position during visual search. Cereb Cortex, in press.

Viswanathan P, Nieder A (2013) Neuronal correlates of a visual "sense of number" in primate parietal and prefrontal cortices. Proc Natl Acad Sci U S A 110:11187-11192. CrossRef Medline

Viswanathan P, Nieder A (2015) Differential impact of behavioral relevance on quantity coding in primate frontal and parietal neurons. Curr Biol 25:1259-1269. CrossRef Medline 